\title{
USO DE BOTS Y ALGORITMOS PARA AUTOMATIZAR LA REDACCIÓN DE NOTICIAS: PERCEPCIÓN Y ACTITUDES DE LOS PERIODISTAS EN ESPAÑA
}

\author{
Automated-content generation using news-writing \\ bots and algorithms: Perceptions and attitudes \\ amongst Spain's journalists
}

\author{
José-Miguel Túñez-López, Carlos Toural-Bran y Santiago Cacheiro-Requeijo
}

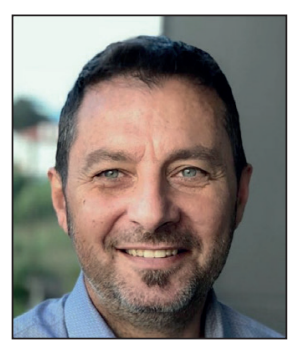

José-Miguel Túñez-López es doctor en Periodismo por la Universidad Autónoma de Barcelona y profesor de Comunicación organizacional y de Estrategias de comunicación en el Departamento de Ciencias de la Comunicación de la Universidade de Santiago de Compostela (USC). Consultor y miembro del grupo de investigación Novos Medios de la USC, es director del programa de Postgrado en Comunicación e Información Contemporánea. Posee un Premio Nacional de Periodismo Reina Sofía.

https://orcid.org/0000-0002-5036-9143

miguel.tunez@usc.es

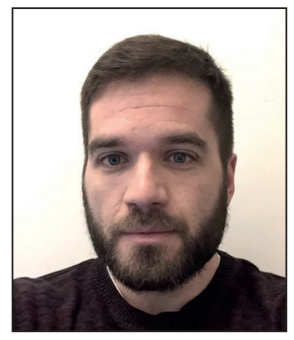

Carlos Toural-Bran es doctor en Ciencias de la Comunicación por la Universidade de Santiago de Compostela (USC) y profesor de Multimedia y Nuevos formatos y Cibercultura en el Departamento de Ciencias de la Comunicación de la misma universidad. Vicedecano de la Facultad de Ciencias de la Comunicación de la USC y periodista, desde 2006 forma parte del grupo de investigación Novos Medios, del que es secretario. Es director de la Revista de la Asociación Española de Investigación de la Comunicación.

https://orcid.org/0000-0002-0961-3925

carlos.toural@usc.es

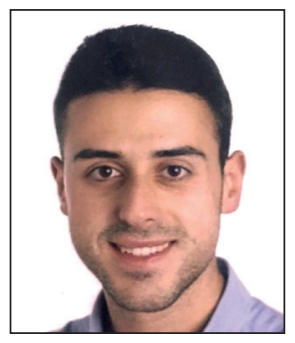

Santiago Cacheiro-Requeijo es periodista especializado en generación automatizada de contenidos y nuevas tecnologías. Ha investigado el tema de la robotización en la producción de noticias en varios trabajos profesionales.

https://orcid.org/0000-0003-3867-9250

santiagocrequeijo@gmail.com

Universidade de Santiago de Compostela

Facultade de Ciencias da Comunicación Avenida Castelao, s/n. 15782 Santiago de Compostela (A Coruña), España

\section{Resumen}

Algoritmos, bots y automatización en la elaboración de noticias. Este conjunto de conceptos, elementos y procesos afecta a la profesión periodística que vive momentos convulsos donde la inteligencia artificial (IA) está empezando a ocupar un terreno tradicionalmente dominado por el factor humano. En esta investigación elaboramos un mapa mundial de medios, agencias y empresas que recurren a la automatización en la elaboración de información. Además, definimos la percepción sobre este fenómeno en España a través de una encuesta a colegios profesionales, asociaciones y sindicatos donde 366 periodistas dibujan el estado de la cuestión con sus respuestas. Los principales resultados muestran cómo existe un desconocimiento profundo por parte de los periodistas españoles de la influencia directa de la IA en la profesión. La automatización de noticias no deja de ganar terreno tal y como demuestran las 50 iniciativas catalogadas a nivel mundial en el mapa elaborado.

\section{Palabras clave}

Inteligencia artificial; Automatización; Bots; Elaboración de noticias; Profesión periodística; Periodistas; Periodismo digital. 


\begin{abstract}
Algorithms, bots and automation for news articles generation. The combination of these concepts, elements and processes is affecting journalism as a profession, which is going through a particularly tumultuous period as artificial intelligence (Al) begins to occupy a field traditionally dominated by the human factor. This research provides a world map featuring media, agencies and companies that use automation to generate information. Moreover, we looked into the perception of this phenomenon in Spain through a survey with chartered professional associations, associations of journalists and unions where 366 journalists provided a picture of the current situation within the country. Our findings prove that there is a deep lack of understanding amongst Spanish journalists about how Al can have a direct impact on journalism as a profession. Automated journalism will not stop gaining ground, as evidenced by 50 projects featured on our media world map.
\end{abstract}

\title{
Keywords
}

Artificial intelligence; Automation; Bots; Newsmaking; Journalistic profession; Journalists; Online journalism.

Túñez-López, José-Miguel; Toural-Bran, Carlos; Cacheiro-Requeijo, Santiago (2018). "Uso de bots y algoritmos para automatizar la redacción de noticias: percepción y actitudes de los periodistas en España". El profesional de la información, v. 27, n. 4, pp. 750-758.

https://doi.org/10.3145/epi.2018.jul.04

\section{Introducción}

La inteligencia artificial supuso en los inicios cambiar las rutinas del periodista automatizando funciones de búsqueda, clasificación o tratamiento de la información y ha comenzado a instalarse ya en sus tareas de redacción de noticias. EI uso de bots ${ }^{1}$ para generar texto

"es la cima de un proceso de décadas de automatización en las redacciones" (Lindén, 2017)

que comenzó al final de la década de los 80 del siglo XX. Los primeros pasos se dieron con la informatización de redacciones que eliminaban la transmisión de noticias de agencia por cable y en papel, cambiaban las máquinas de escribir por ordenadores y significaban el fin de las cintas de carrete abierto en la radio o abrían paso a la edición virtual en la televisión.

Los cambios han continuado en diferentes fases a lo largo de las últimas tres décadas, tanto en infraestructuras como en aparición de nuevos productos y nuevos soportes, en los modos de impresión y de distribución de señal y de productos, en la organización de trabajo, en el equipamiento e incluso en los modos de enfoque del periodismo con el auge del periodismo de precisión y, después, del periodismo basado en la interpretación y presentación de datos asociado a los big data (datos masivos).

La automatización de noticias es la aplicación de la inteligencia artificial (IA) al newsmaking y supone la identificación de rutinas reiteradas que pueden ser codificadas en algoritmos que generen productos similares a los que se obtienen de esa misma tarea ejecutada por humanos. Esa similitud en los resultados se logra gracias a los generadores de lenguaje natural, es decir, programas capaces de transformar datos computacionales estructurados en lenguaje humano comprensible por la audiencia (Dörr, 2016; Marconi; Siegman, 2017).

La automatización de funciones y sus cambios en los entornos laborales es una realidad que afecta globalmente a todos los ecosistemas de trabajo, no sólo al periodismo. En este caso sin embargo abre debates que van más allá de la sustitución del individuo por la máquina ya que se trasladan a la deontología, la veracidad de contenidos y a la creación de nuevas esferas de control sobre la información que se publica.

El periodismo plenamente automatizado no trabaja directamente sobre la realidad sino sobre una realidad codificada en datos sobre la que actúan los algoritmos, conjuntos ordenados y finitos de normas específicas que aplicados a un problema conducen a su resolución. El debate gira en torno al proceso de elaboración de las bases de datos y los criterios de selección y jerarquía utilizados, pero también se adentra en analizar la capacidad de la IA para llegar a sustituir la parte cognitiva del trabajo periodístico, en la posibilidad de adulteración intencional del proceso para generar noticias falsas y en las decisiones para determinar reglas de construcción del algoritmo.

Como indican Marconi y Siegman (2017), el debate sobre aspectos éticos hace surgir reclamos para que la IA garantice la transparencia de los programas de automatización porque

"Ios lectores tienen derecho a entender cómo es usada la inteligencia artificial, así como las decisiones que se toman en términos comprensibles, sin tecnicismos"

(Hansen et al., 2017).

Los estudios sobre el impacto de la automatización en el periodismo aún son escasos, aunque hay trabajos referenciales que sirven para delimitar su alcance y que se han tomado como fuente para la realización de esta investigación. Entre estas aportaciones, las de Kim et al., 2007; Matsumoto et al., 2007; Van-Dalen, 2012; Clerwall, 2014; Edge, 2014; KarIsen; Stavelin, 2014; Latar, 2014; Napoli, 2012; Lecompte, 2015; Dörr, 2016; Graefe, 2016; Fanta, 2017; Hansen et al., 2017; Lindén, 2017; Marconi; Siegman, 2017.

La automatización de labores rutinarias, en opinión de Lindén, refuerza la eficiencia y la satisfacción laboral, liberando tiempo al periodista y abriendo campo a nuevas formas de 
trabajo con ordenadores. Advierte, sin embargo, cómo parte de las reglas periodísticas para construir los algoritmos se basan en decisiones "inconsistentes y flexibles" (Lindén, 2017) que obligan a mantener en el proceso de producción de contenidos "juicios creativos e intuitivos" que, al menos de momento, no están en el campo de acción robotizada y que se mantendrán a salvo si se estimula la capacidad del periodista de "destilar ideas" es decir, hacer propuestas que agreguen valor al proceso de construir noticias.

Las investigaciones coinciden en que la profesión no corre peligro aunque, como señala Graefe (2016), el futuro se contempla con cambios y ajustes al tiempo que se describe como un matrimonio entre los periodistas y las máquinas. Algunos estudios, sin embargo, evidenciaron que los materiales informativos elaborados mediante inteligencia artificial resultaban más creíbles que los elaborados por humanos. Clerwall (2014) analizó las reacciones de la audiencia a noticias escritas por una persona y a las elaboradas por una máquina y concluyó que para el público no había diferencias importantes entre ambos textos. El digital news report 2017 del Reuters Institute y la University of Oxford (Newman et al., 2017) refuerza esta tendencia al ofrecer datos de preferencias del público sobre noticias seleccionadas por editores humanos o por algoritmos. En general, un 54\% se decanta por la selección automatizada frente al $44 \%$ que opta por la realizada por humanos. Cuando se revisaban los datos de los menores de 35 años la preferencia por la propuesta informativa realizada por un robot aumentaba hasta el $64 \%$.

Los estudios sobre el impacto de la automatización en el periodismo aún son escasos

En la revisión de bibliografía científica no se han encontrado investigaciones significativas sobre la automatización de noticias en España. Fanta (2017) refiere en su estudio de agencias europeas que la Agencia Efe no ha considerado aún su utilización, aunque señala que alguna de sus delegaciones sí trabaja con pequeños sistemas de tratamiento automatizado de datos. También se han identificado experiencias pioneras como la de Vocento al crear información de servicio sobre playas o estaciones de esquí en un proyecto para automatizar la actualización de contenidos denominado Medusa, o empresas como Narrativa que está entre las pioneras en orientarse a la elaboración de informes de temática deportiva en tiempo real para el ámbito editorial.

\section{Materiales y métodos}

La investigación que sustenta este artículo se orientó a identificar las empresas, los medios y las agencias que ya aplican algoritmos para generar informáticamente contenidos informativos elaborados del mismo modo en que lo haría un redactor y a pulsar cómo la profesión periodística se enfrenta a un proceso universal que no aparece situado como referencia destacada cuando se abordan las tendencias de futuro sobe inteligencia artificial e internet de las cosas, pero que afecta a las bases y a los modos de trabajo de la profesión. Es una investigación aplicada que se puede considerar exploratoria ya que no tiene como fin producir unas conclusiones generalizables sino sedimentar conocimiento alrededor de un problema de investigación que necesita de aportaciones científicas para avanzar en su definición.

Este trabajo se apoya metodológicamente en la encuesta como compendio de fases que, de manera organizada, busca recoger datos. La encuesta, en palabras de García-Ferrando (citado en: Casas-Anguita; Repullo-Labrador; Donado-Campos, 2003), es la técnica que, mediante procedimientos estandarizados de investigación recoge datos de una muestra de casos que resulta representativa de una población o universo más amplio, del que se pretende explorar, describir, predecir y/o explicar una serie de características.

El cuestionario, tal y como apunta Meneses es:

"el instrumento estandarizado que utilizamos para la recogida de datos durante el trabajo de campo de algunas investigaciones cuantitativas, fundamentalmente, las que se llevan a cabo con metodología de encuesta" (Meneses, 2016).

Para su diseño se ha optado por un modelo híbrido de preguntas, algunas con escalas tipo Likert, donde se intercalan preguntas de respuesta abierta con otras cerradas. En las abiertas el interés principal a nivel estratégico es obtener una respuesta de tipo reflexiva donde la carga experiencial personal, así como el bagaje cultural y profesional resultan muy relevantes para la investigación. Las preguntas cerradas se emplean para obtener un posicionamiento de intensidad con respecto a una variable usando escalas de puntuación y en casos en los que las alternativas propuestas en las respuestas eran limitadas.

Con el objetivo de favorecer un número elevado de respuestas se ha optado por un cuestionario corto de diez preguntas a través de las que se aborda el conocimiento y la percepción sobre la automatización de contenidos en la profesión periodística en España en los siguientes términos:

- Valoración sobre la aplicación de la inteligencia artificial al periodismo.

- Valoración de una redacción integrada por bots y algoritmos.

- Identificación de medios que los periodistas saben que recurren o prevén recurrir a la automatización.

- Percepción de cómo la automatización de contenidos afecta a su calidad, el empleo, la imagen de la profesión y el coste de producción de contenidos.

- Actitud de los profesionales ante la automatización por áreas informativas.

En la construcción de la muestra hemos considerado un universo compuesto por los Colegios Oficiales de Periodistas de España (Andalucía, Castilla y León, Cataluña, Galicia, La Rioja, Murcia y Euskadi), la Federación de Asociaciones de la Prensa (FAPE) y de modo individual se ha contactado con 48 de sus 51 asociaciones regionales federadas) y la Federación de Sindicatos de Periodistas (FeSP) a través de los Sindicatos de Periodistas de Andalucía (SPA), Catalunya (SPC), Illes Balears (SPIB), La Rioja (SPIR), Madrid (SPM), Galicia (SXG) y la Unión de Profesionales de la Comunicación de Canarias (UPCC). 
Con su colaboración, se ha puesto a disposición de sus asociados el cuestionario, a través de un enlace web desde la plataforma SurveyMonkey elegida por criterios de usabilidad en dispositivos móviles para conseguir un mayor número de respuestas.

https://es.surveymonkey.com

Al tratarse de un cuestionario online, se incorporó como pregunta obligatoria la casilla de correo electrónico para poder verificar los registros. Se validaron 366 respuestas entre el 5 de febrero y el 5 de marzo de 2018.

Con este diseño metodológico hemos abordado de manera directa la hipótesis de partida: los profesionales españoles desconocen el grado de implantación de la automatización en la elaboración de contenidos en medios de comunicación a nivel nacional e internacional y dudan de su utilidad en términos operativos en relación con la creación informatizada de las noticias.

\section{Análisis y resultados}

\subsection{Identificación de medios y empresas que recu- rren a la automatización de la elaboración de noticias}

En el mapa mundial de medios y empresas que recurren de modo significativo a la elaboración automatizada de noticias se pueden señalar 26 medios (16 medios periodísticos y 13 agencias de noticias) y 21 empresas, principalmente de América del Norte, Europa, China y Japón. Por países, las mayores concentraciones se dan en Estados Unidos, Alemania y Reino Unido.

\section{6 medios, 13 agencias y 21 empresas} lideran a nivel mundial el escenario de la automatización de noticias

Las primeras menciones de inteligencia artificial se remontan a la mitad del siglo XX. Se cita por primera vez en un evento académico en 1955 (McCarthy et al., 2017), cinco años después del Turing test (Turing, 1950) para medir la capacidad de las máquinas para asumir comportamientos humanos. Las referencias concretas a contenidos elaborados automáticamente se centran en textos de información del tiempo (Meehan, 1977; Glahn, 1970) o, ya en la década de los 90, de deportes y de economía (Meehan, 1977). En estos años 90 irrumpen las soluciones ofertadas por empresas como Bloomberg L.P., una compañía estadounidense de software financiero, datos y noticias y que tiene entre sus clientes a Thomson Reuters, Dow Jones Newswires y New York Financial Press (Winkler, 2012).

El auge del periodismo de datos y de los programas de minería de datos dieron el impulso a la automatización de informaciones que comenzó a analizarse como robot journalism (Lee; Kim, 1998) o automated journalism y más recientemente como periodismo cognitivo (Lindén, 2017). Hay coincidencia en considerar que la automatización masiva la inicia Associated Press con Automated Insights y Zacks Investment Research, en 2014, al generar 3.000 noticias sobre ganancias corporativas, aunque le precede el algoritmo Quakebot de Los Angeles Times que usa datos del United
States Geological Survey para elaborar noticias sobre movimientos sísmicos y lanza informaciones elaboradas a partir de una plantilla previa que se publican directamente si el temblor es menor de 6 grados.

The Washington Post (con los Juegos Olímpicos de Río 2016) y Associated Press (2014) han reforzado la automatización de contenido a gran escala centrándose principalmente en deportes y finanzas, pero pequeñas redacciones como Local Labs, Hoodline y Hereford Times (Wang, 2018; Lindén, 2017; Sandle, 2018) también están empleando algoritmos para la cobertura de servicios y sucesos locales que personalicen sus informaciones.

En el mapa actual se han catalogado las iniciativas que se relacionan (Lecompte, 2015; Dörr, 2016; Mullin, 2016; Cid, 2017; Lindén, 2017; Goichman, 2017; Villar-Gutiérrez, 2017; Villareal, 2017; Graefe, 2016; Fanta, 2017; Muñoz, 2017; Sandle, 2018; Wang, 2018; Fuchs, 2018):

\section{Medios}

a) Alemania:

- Berliner Morgenpost: información sobre la contaminación por partículas en Berlín.

- Der spiegel: automatización en pruebas aplicada a información deportiva.

- Finanzen100.de: información financiera.

- Fupa.net (Antes, Fubanews): información de deportes.

- Goekick.com: información de deportes locales.

- Handelsblatt: información de deportes, tiempo y economía.

- Neue Osnabrücker Zeitung: información de deportes.

- RadioHamburgFussiFreunde: información de deportes.

- Rheinfussball: información de deportes locales.

- Weserkurier: información de deportes locales.

b) China: South China morning post: gestiona contenidos y estrategias con datos obtenidos del comportamiento de sus lectores.

c) España. Vocento. Proyecto Medusa: información de servicios (infoPlayas e infoEsquí).

d) Estados Unidos:

- BigTenNetwork: información de deportes y finanzas.

- Forbes: información de finanzas y deportes.

- GameChanger: información de finanzas y deportes.

- Hoodline: elaboración de noticias muy locales en San Francisco.

- Local Labs: información de eventos locales.

- Los Angeles Times: información meteorológica (Quakebot) e informes de homicidios en tiempo real (Homicide report).

- ProPublica: análisis de la calidad de la educación estadounidense (Opportunity gap).

- Quartz: desarrolla robots conversacionales e inteligencia artificial en su Quartz Bot Studio para ayudar a periodistas.

- Sports illustrated.

- The New York Times: información política y deportiva. Robots conversacionales.

- The Washington Post: artículos sobre política y deportes gracias a su aplicación Heliograf.

- Yahoo!: información de deportes. (Yahoo!Sports). 
e) Francia: Le monde: información política en las elecciones.

f) Japón: The Shinano mainichi shimbun (anuncio propio, en enero 2018): solución automatizada para sumarizar noticias de forma instantánea.

g) México: El financiero (lo anuncia en algunos de sus textos).

h) Reino Unido:

- Hereford Times.

- MeteoGroup: pronósticos meteorológicos.

- Telegraph and Argus.

- The Derby Telegraph.

\section{Agencias}

- China: Xinhua: responsable del desarrollo de Jia Jia, un robot humanoide.

- Estados Unidos: Associated Press: información de finanzas y deporte (mediante Wordsmith, de Automated Insights).

- Holanda: ANP: simplificación de noticias para su servicio Kids Feed.

- Noruega: NTB: información de deportes y finanzas.

- Portugal: Lusa: pruebas de información política en elecciones parlamentarias 2015.

- Reino Unido: Press Association y Reuters: principalmente finanzas y deportes.

- DPA (Alemania), STT (Finlandia), AFP (Francia), APA (Austria), Ritzau (Dinamarca) y TT (Suecia) referenciadas por Fanta (2017) como agencias que admiten transmitir contenidos generados por bots, aunque no concreta temática.

Empresas generadoras de servicios de automatización de noticias

- Alemania: 2txt, Aexea, AX Semantics, Retresco, Text-on y Textomatic.

- China: Tencent.

- España: Narrativa.

- Estados Unidos: Automated Insights, Bloomberg, Linguastat, Narrative.

- Science, OnlyBoth, Yseop (con representación en Francia y en Reino Unido).

- Francia: Labsense, Syllabs.

- Israel: Articoolo.

- Japón: Fujitsu (anuncio propio, en enero 2018).

- Reino Unido: Arria.

- Suecia: United Robots.

3.2. Grado de conocimiento de los procesos de automatización por la profesión periodística en España

La automatización robótica para elaborar contenidos informativos no parece estar presente en la agenda de preocupaciones de los periodistas españoles. Nueve de cada diez $(86,9 \%)$ aseguran que no conocen ningún caso. De los que dicen que sí han oído hablar de medios que recurren a un algoritmo para producir contenidos informativos, tan sólo tres aciertan a registrar una referencia válida al señalar a Los Angeles Times y la agencia de noticias Reuters entre las entidades que ya emplean bots para realizar tareas periodísticas.

Cuando se profundiza en el tema y se pregunta por algún medio que tenga en proyecto o esté trabajando para recurrir a la automatización en la elaboración de contenidos los porcentajes aumentan: el 93,98\% dice que no sabe de ningún caso y las referencias a cabeceras o a empresas concretas se limitan a dos: Google y The New York Times, además de una acertada indicación de la empresa Narrativa.

\section{Siete de cada diez periodistas consideran que no sería posible crear una redacción completamente robotizada}

Siete de cada diez periodistas $(68,7 \%)$ consideran que no sería posible crear una redacción completamente robotizada y el $61,2 \%$ afirma que en todo caso las noticias creadas a través de inteligencia artificial sólo serían complementarias a las que elaborasen los redactores humanos.

\subsection{Percepción y actitud de los periodistas en España}

Para indagar en la percepción de la profesión sobre la sustitución de redactores por bots se presentó una lista cerrada de 8 respuestas. Se pedía a los periodistas que indicaran cuál sería la posible aplicación o impacto que tendría el hecho de aplicar la robotización de las redacciones a la creación de contenidos. La mayoría se inclina por decir que sólo sería aplicable a la distribución o la difusión de las noticias, pero no a su elaboración $(21,3 \%)$. Uno de cada cuatro se decanta por elegir que el impacto sería sobre el proceso de distribución del producto informativo (12\%) o para lograr nuevas formas de llegar a los públicos (12,5\%). Para un 30\% sería un modo de trabajar mejor con bases documentales (11,5\% afirma que sería de utilidad en tareas de documentación y el $20,8 \%$ en tareas de tratamiento de datos). Los

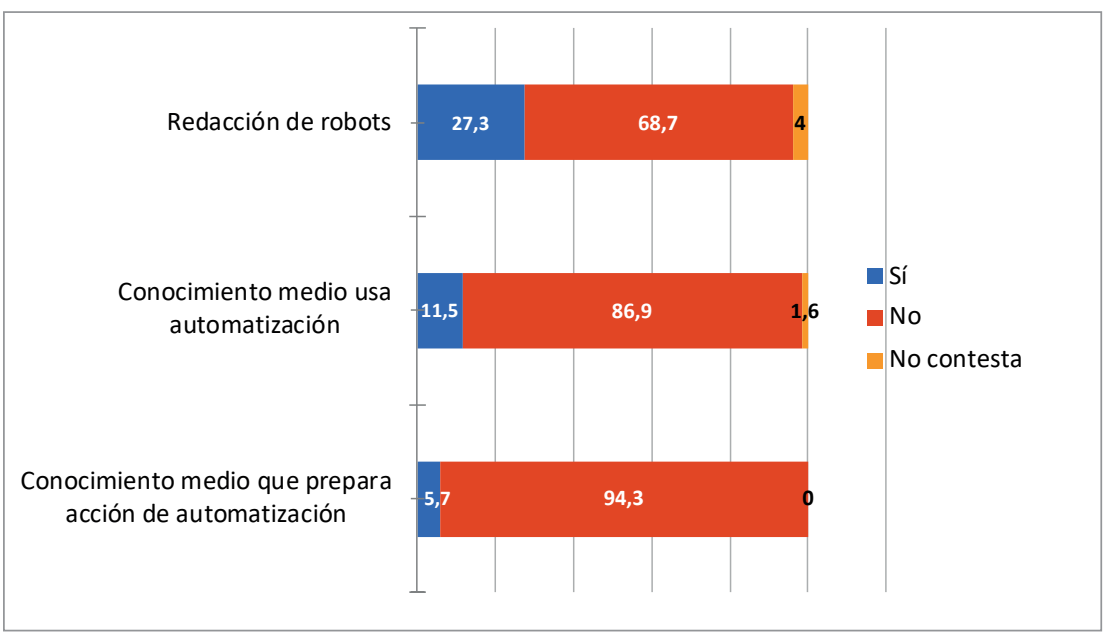

Gráfico 1. Grado de conocimiento de los procesos de automatización 


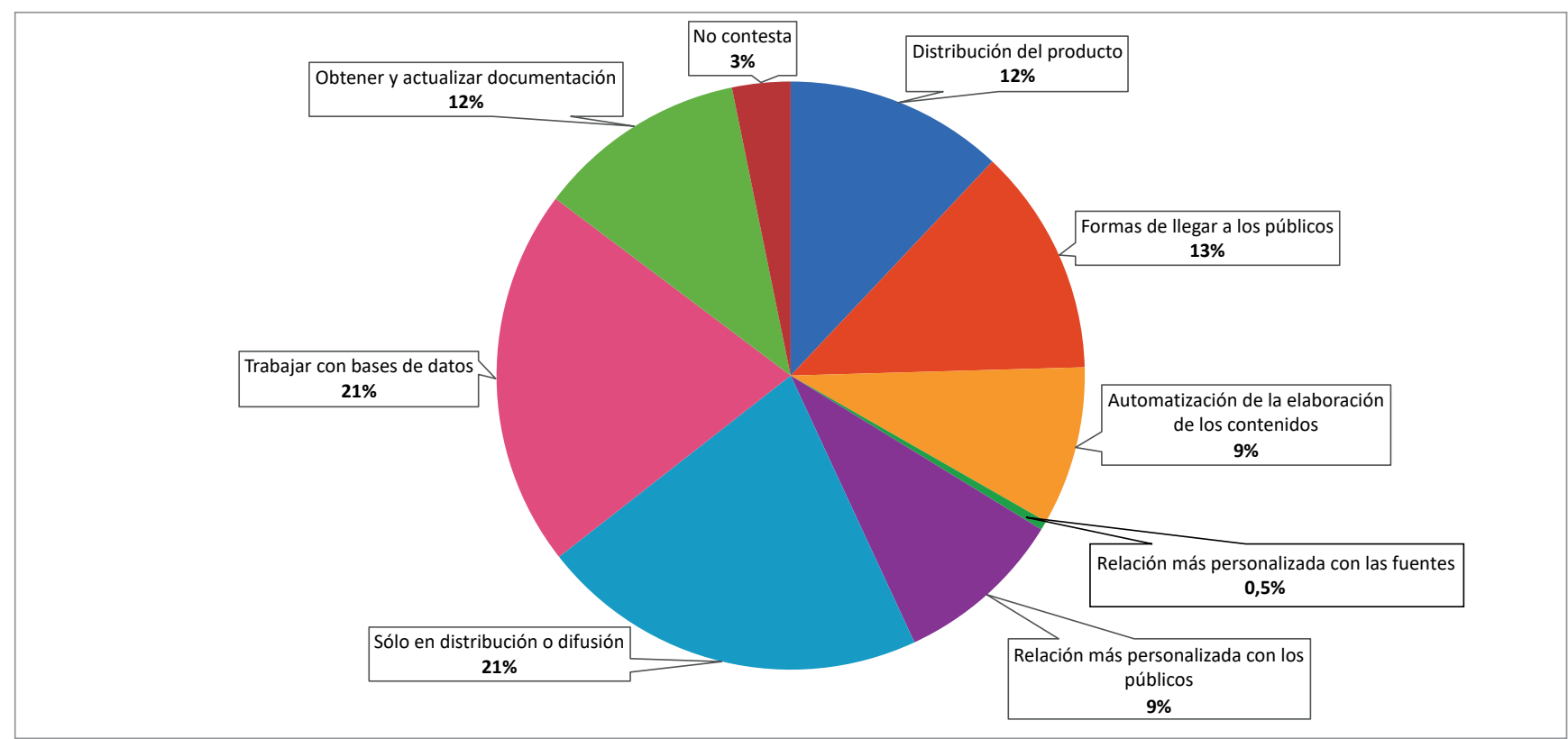

Gráfico 2. Aplicaciones de la robotización para crear contenidos

indicadores con menores porcentajes de adhesión son los que están directamente relacionados con lograr a través de la inteligencia artificial una relación más personalizada con las fuentes $(0,5 \%)$ y en la creación automatizada de textos o contenidos noticiosos $(8,7 \%)$ o una relación más personalizada con los públicos $(9,3 \%)$.

Entre los periodistas españoles no hay conciencia clara de que la generación de noticias a través de algoritmos ha empezado a ser una realidad

La medición de la percepción de la profesión sobre la generación automatizada de contenidos informativos se orientó, también, a determinar si hay diferencias en función del tema que se aborde en la noticia. En una escala de 0 (total-

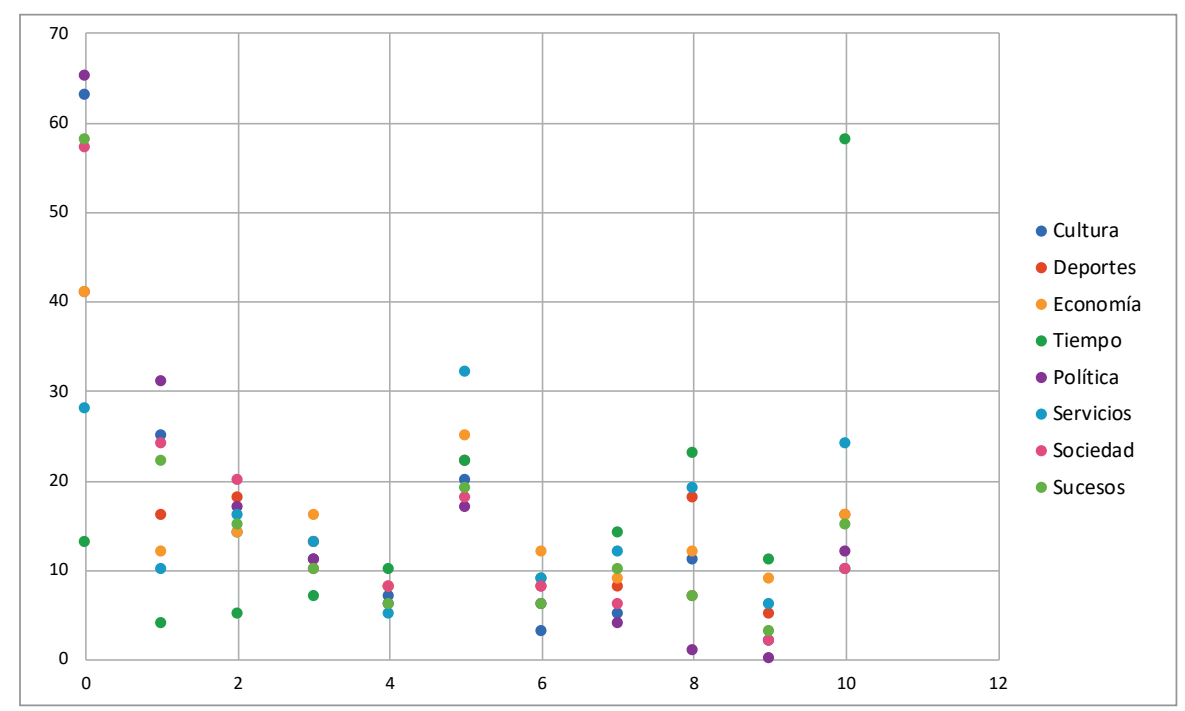

Gráfico 3. Temas en los que los periodistas ven factibles las noticias generadas por bots mente imposible) a 10 (totalmente posible), los periodistas afirman que la actualidad meteorológica (6,82 de media) y de servicios $(4,86)$ podrían llegar a ser una actividad realizada por robots. El resto de las áreas concentran respuestas que se acercan a negar la posibilidad de robotizar: Economía $(4,12)$, Deportes $(3,97)$, Sucesos $(3,16)$ y, principalmente, Sociedad $(2,82)$, Cultura $(2,77)$ y Política $(2,35)$.

Hay una alta dispersión de respuestas que hace interesante revisar los resultados atendiendo a la moda y a la polarización en los extremos de la tabla. En la información del Tiempo, la moda es 10 (totalmente posible), pero en Servicios es 5 (la mitad de la escala). Ordenados de modo ascendente según el número de respuestas acumuladas, en Deportes, Economía, Sociedad, Sucesos, Cultura y Política, la moda es cero (0). Agrupando todas las respuestas de los extremos de la tabla (10 y 9 frente a 0 y 1 ) para cada una de las opciones propuestas, las respuestas de periodistas que consideran totalmente imposible que se automaticen contenidos son más del doble $(2,5)$ que las afirmaciones de los que lo ven totalmente posible. En Política, por cada respuesta que acepta la posibilidad de robotización hay ocho que la ven inviable.

Cuando se proyecta la automatización sobre aspectos concretos de la profesión, la valoración más positiva es sobre el modelo de negocio. El interés de las empresas estaría, para el $100 \%$ de los entrevistados, en aumentar la productividad disminuyendo la inversión en el proceso de generar información. La mayoría cree que los costes de producción se abaratarían (7,25 en 


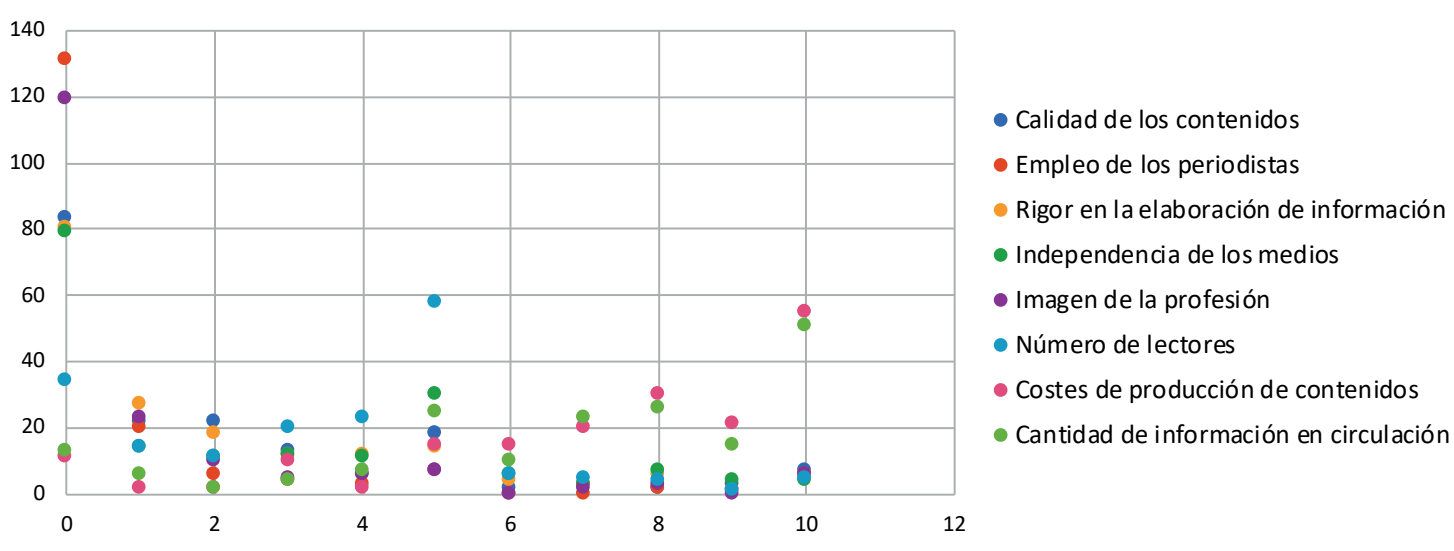

Gráfico 4. Percepción del impacto de la automatización de contenidos en la profesión

una escala de 0 a 10) y aumentaría la cantidad de la información $(6,86)$ que se pondría en circulación. No se augura, sin embargo, que una producción más económica y una oferta mayor redundase en un aumento del número de lectores (3,53 sobre 10$)$.

Es cuando menos curioso que al evaluar el impacto sobre aspectos de producción, de fusión y de elaboración de noticias, los elementos estrechamente vinculados a los contenidos no encabecen el listado. Para los periodistas, robotizar la redacción informativa impactaría sobre todo en el empleo $(0,97$ sobre 10 en una escala en la que 0 equivale a "muy negativamente") y en la imagen global de la profesión ante la sociedad $(1,18)$. Los profesionales atribuyen un mayor impacto negativo de la robotización sobre el empleo de los periodistas que sobre elementos directamente vinculados con la esencia de la profesión como la independencia $(2,51)$, la calidad $(2,01)$ y el rigor $(2,51)$.

Ninguno de los 366 periodistas encuestados aludió a la necesidad de repensar su intervención personal en el proceso de creación de la noticia

\section{Conclusiones}

De los resultados se deriva que entre los profesionales del periodismo aún no hay conciencia clara de que la generación de noticias a través de algoritmos ha dejado de ser una posibilidad para ser una realidad, con lo que queda confirmada la hipótesis de partida de manera positiva. Incluso se desconoce que algunos medios y agencias internacionales ya han sustituido a sus redactores por aplicaciones informáticas para elaborar los contenidos que transmiten a sus audiencias. Seguramente en esta percepción influye el hecho de que en España sólo se hayan identificado hasta el momento tímidos intentos de producir contenidos a través de bots. Los casos de automatización en nuestro país no van más allá de iniciativas concretas en servicios online de información del tiempo o servicios o en propuesta de empresas que genéricamente ofrecen la elaboración automatizada de contenidos.
Estados Unidos, Alemania y Reino Unido concentran la mayoría de las iniciativas mediáticas o empresariales que usan u ofertan información generada informáticamente. Los casos más significativos permiten dibujar un mapa mundial con 16 medios informativos, 13 agencias nacionales de noticias y 21 empresas. Resulta llamativo que, entre los periodistas que participan en el estudio, sea porcentualmente insignificante el caso de aquellos que son capaces de proponer referencias de algún medio que ya esté recurriendo a bots para automatizar la generación de informaciones.

La aplicación directa de inteligencia artificial en la elaboración de contenidos por máquinas se ve más como un cambio en las rutinas productivas del periodista que como una alteración de todo el proceso de newsmaking en el que el periodista llega a desaparecer. Los posibles efectos se vinculan con estrategias empresariales para reducir los costes de producción o para controlar la orientación de los contenidos. También se alude a una posible aplicación para que las organizaciones elaboren más informaciones para remitir a los medios como propuesta de inclusión en sus temarios (publicity o free press).

Los flujos de comunicación con las audiencias están cambiando y el fortalecimiento de las redes sociales como nuevo soporte de transmisión y nuevo escenario de encuentro con los públicos acuña un modo de relación bidireccional simétrica que caracteriza la gestión comunicativa y de relaciones del siglo XXI. No obstante, la inteligencia artificial y la automatización de contenidos no se vislumbran como un elemento de transformación y cambio o de, al menos, dinamización o alteración en las relaciones de los informadores o los medios y con audiencias. Sólo uno de cada diez periodistas considera que la IA permitirá una relación más personalizada con los públicos, siguiendo la misma línea que la personalización de contenidos que se identifica como elemento diferenciador de los nuevos modos de entender el marketing y de gestionar la comunicación de las organizaciones en esta segunda década del siglo XXI.

El periodismo no escapa al proceso de automatización laboral global como consecuencia del desarrollo de la inteligencia artificial, la robótica y las nuevas tecnologías de la comunicación, pero se sostiene que las tareas que requie- 
ren de habilidades cognitivas son más difíciles de encuadrar en acciones estandarizadas reproducibles por una máquina.

No se evaluaron en esta fase de la investigación las actitudes de respuesta de la profesión para mitigar los impactos negativos y para promover los que pudieran resultar beneficiosos. Pero es importante destacar que ninguno de los 366 periodistas participantes aludió en las respuestas abiertas a la necesidad de repensar su intervención personal en el proceso de creación de la noticia potenciando, como se destaca en otros estudios sobre automatización, la contribución de la intervención de la persona a la generación de valor identificable en el texto a través de la singularización de propuestas o enfoques. Es decir, intentar esquivar el algoritmo enfatizando el componente intelectual que convierta la elaboración del texto en un proceso alejado de decisiones mecánicas y de la redacción reiterativa en sus estructuras y en su terminología.

\section{Agradecimientos}

Los resultados de este artículo forman parte de las actividades de investigación promovidas a través de la Red Internacional de Investigación de Gestión de la Comunicación (ED341D R2016/019 Xescom), apoyada por la Consellería de Cultura, Educación e Ordenación Universitaria de la Xunta de Galicia; de las tareas exploratorias del proyecto del Programa estatal de Fomento de la Investigación Científica y Técnica de Excelencia, subprograma estatal de Generación de conocimiento del ministerio de economía y competitividad de España sobre Indicadores de gobernanza, financiación, rendición de cuentas, innovación, calidad y servicio público de las RTV europeas aplicables a España en el contexto digital (Referencia CSO2015-66543-P); y de la fase de análisis de resultados del proyecto Usos y preferencias informativas en el nuevo mapa de medios en España: modelos de periodismo para dispositivos móviles (Referencia: CSO2015-64662C4-4-R), financiado por el Ministerio de Economía y Competitividad y por el Fondo Europeo de Desarrollo Regional. Se tomó una muestra de indicadores del objeto de ambos proyectos para la realización de la encuesta a los profesionales de la información.

\section{Nota}

1. Aféresis de "robots". Son programas informáticos constituidos por conjuntos de reglas que se ejecutan en internet de manera repetitiva efectuando acciones de tipo recurrente.

\section{Referencias}

Casas-Anguita, Juana; Repullo-Labrador, José-Ramón; Donado-Campos, Juan (2003). "La encuesta como técnica de investigación: elaboración de cuestionarios y tratamientos estadísticos de los datos". Atención primaria, v. 31, n. 8, pp. 527-538.

https://goo.gl/xM9DwC

https://doi.org/10.1016/S0212-6567(03)70728-8

Cid, Guillermo (2017). "Este robot reemplazará a los periodistas (empezando por los deportivos)". El confidencial, 31 agosto. https://www.elconfidencial.com/tecnologia/2017-08-31/ goles-robots-noticias-narrativa-startup_1436100
Clerwall, Christer (2014). "Enter the robot journalist". Journalism practice, v. 8, n. 5 , pp. 519-531.

https://doi.org/10.1080/17512786.2014.883116

Dörr, Konstantin-Nicholas (2016). "Mapping the field algorith, journalism". Digital journalism, v. 4, n. 6, pp. 700-722. https://goo.gl/2KauZr https://doi.org/10.1080/21670811.2015.1096748

Edge, Abigail (2014). “Ophan: Key metrics informing editorial at The Guardian". Journalism.co.uk, Dec. $2^{\text {nd }}$. https://www.journalism.co.uk/news/how-ophan-offersbespoke-data-to-inform-content-at-the-guardian/s2/a563349

Fanta, Alexander (2017). Putting Europe's robots on the map: Automated journalism in news agencies. University of Oxford; Reuters Institute for the Study of Journalism. https://goo.gl/wBfuQs

Fuchs, Martina (2018). "Robo-reporters: The end of the story for journalism?". Huffington post, Jan. $17^{\text {th }}$. https://goo.gl/kGV8qu

Glahn, Harry R. (1970). "Computer worded forecasts". Bulletin of the American Meteorological Society, v. 51, n. 12, pp. 1126-1132.

https://goo.gl/4Juqff

https://doi.org/10.1175/1520-0477(1979)060\%3C0004:CW F\%3E2.0.CO;2

Goichman, Rafaella (2017). "Written by a robot: Will algorithms kill journalism?". Haaretz, Febr. $15^{\text {th }}$.

https://www.haaretz.com/israel-news/business/1.771758

Graefe, Andreas (2016). Guide to automated journalism. Tow Center for Digital Journalism, Jan. $7^{\text {th }}$.

https://www.cjr.org/tow_center_reports/guide_to_ automated_journalism.php

Hansen, Mark; Roca-Sales, Meritxell; Keegan, Jonathan M.; King, George (2017). Artificial untelligence: Practice and implications for journalism. Collumbia University Libraries; Tow Center for Digital Journalism.

https://doi.org/10.7916/D8X92PRD

Karlsen, Joakim; Stavelin, Eirik (2014). "Computational journalism in Norwegian newsrooms". Journalism practice, v. 8, n. 1 , pp. $34-48$.

https://doi.org/10.1080/17512786.2013.813190

Kim, Jong-Hwan; Lee, Kang-Hee; Kim, Yong-Duk; Kuppuswamy, Naveen-Suresh; Jo, Jun (2007). "Ubiquitous robot: A new paradigm for integrated services". En: 2007 IEEE Intl conf on robotics and automation, pp. 2853-2858. https://doi.org/10.1109/ROBOT.2007.363904

Latar, Noam-Lemelshtrich (2014). "Robot journalists: 'Quakebot' is just the beginning”. Knowledge@Wharton, March $28^{\text {th }}$.

http://knowledge.wharton.upenn.edu/article/will-robotjournalists-replace-humanl-ones

Lecompte, Celeste (2015). "Automation in the newsroom". Nieman reports, Sept, $1^{\text {st }}$.

http://niemanreports.org/articles/automation-in-thenewsroom 
Lee, Sung-Min; Kim, Tai-Yun (1998). "A news on demand service system based on robot agent". En: 1998 Int conf on parallel and distributed systems, pp. 528-532.

https://doi.org/10.1109/ICPADS.1998.741128

Lindén, Carl-Gustav (2017). "Algorithms for journalism: The future of news work". The journal of media innovations, v. 4, n. 1 , pp. 60-76.

https://doi.org/10.5617/jmi.v4i1.2420

Marconi, Francesco; Siegman, Alex (2017). The future of augmented journalism: A guide for newsrooms in the age of smart machines.

https://insights.ap.org/uploads/images/the-future-ofaugmented-journalism_ap-report.pdf

Matsumoto, Rie; Nakayama, Hideki; Harada, Tatsuya; Kuniyoshi, Yasuo (2007). "Journalist robot: Robot system making news articles from real world". En: 2007 IEEE Int/ conf on robotics and automation, pp. 1234-1241.

https://doi.org/10.1109/IROS.2007.4399598

McCarthy, John; Minsky, Marvin; Rochester, Nathaniel; Shannon, Claude (2017). "A proposal for the Dartmouth summer research project on artificial intelligence". Al magazine, v. 27, n. 4, pp. 44-53.

https://doi.org/10.1609/aimag.v27i4.1904

Meehan, James (1977). "Tale-spin, an interactive program that writes stories". En: Procs of the $5^{\text {th }}$ Int joint conf on artificial intelligence, pp. 91-98.

https://www.cs.utah.edu/nlp/papers/talespin-ijcai77.pdf

Meneses, Julio (2016). "El cuestionario. La entrevista”. En: Fàbregues, Sergi; Meneses, Julio; Rodríguez-Gómez, David; Paré, Marie-Hélène. Técnicas de investigación social y educativa. Barcelona, Oberta UOC Publishing, pp. 17-158. ISBN: 9788491163268

http://femrecerca.cat/meneses/files/tecnicas_de_ investigacion_social_y_educativa_2016.pdf

Mullin, Benjamin (2016). "Bloomberg EIC: Automation is "crucial to the future of journalism". Poynter, April $27^{\text {th }}$.

https://www.poynter.org/news/bloomberg-eic-automationcrucial-future-journalism

Muñoz, Carlos (2017). “¿Quieres saber qué implica el auge de los robots para los medios? El \#periodismo en la era de la inteligencia artificial (vol. I)". Medium, 23 junio.

https://medium.com/@VocentoLab/quieres-saberqu\%C3\%A9-implica-el-auge-de-los-robots-para-los-medios$73 a c f b 908615$

Napoli, Philip (2012). "Audience evolution and the future of audience research". International journal on media management, v. 14, n. 2, pp. 79-97.

https://doi.org/10.1080/14241277.2012.675753

Newman, Nic; Fletcher, Richard; Kalogeropoulos, Antonis; Levy, David; Nielsen, Rasmus-Kleis (2017). Reuters institute digital news report 2017. Reuters Institute; University of Oxford. https://goo.gl/dWnxvQ

Sandle, Tim (2018). “Op-Ed: Has this article been written by a robot?". Digital journal, Febr. $3^{\text {rd }}$.

https://goo.gl/Mgfibs

Turing, Alan (1950). "Computing machinery and intelligence". Mind, v. 59, n. 236, pp. 433-460. https://doi.org/10.1093/mind/LIX.236.433

Van-Dalen, Arjen (2012). "The algorithms behind the headlines". Journalism practice, v. 6, n. 5-6, pp. 648-658.

https://doi.org/10.1080/17512786.2012.667268

Villar-Gutiérrez, Miguel-Ángel (2017). “infoPlayas e infoEsquí, dos experimentos de periodismo-robot para los lectores". Medium, 29 marzo.

https://medium.com/@VocentoLab/infoplayas-einfoesqu\%C3\%AD-dos-experimentos-de-periodismo-robotpara-los-lectores-53f42514d533

Villareal, Melissa (2017). "El impacto de la IA y la automatización sobre las noticias". MediaLab press, 31 mayo.

https://medialab.press/impacto-la-ia-la-automatizacionlas-noticias

Wang, Shan (2018). "This hyperlocal news site in San Francisco is reinventing itself with an automated local news wire". NiemanLab, Febr. $5^{\text {th }}$.

http://www.niemanlab.org/2018/02/this-hyperlocalnews-site-in-san-francisco-is-reinventing-itself-with-anautomated-local-news-wire

Winkler, Matthew (2012). The Bloomberg way: A guide for reporters and editors. New Jersey: John Wiley \& Sons. ISBN: 9781118842331

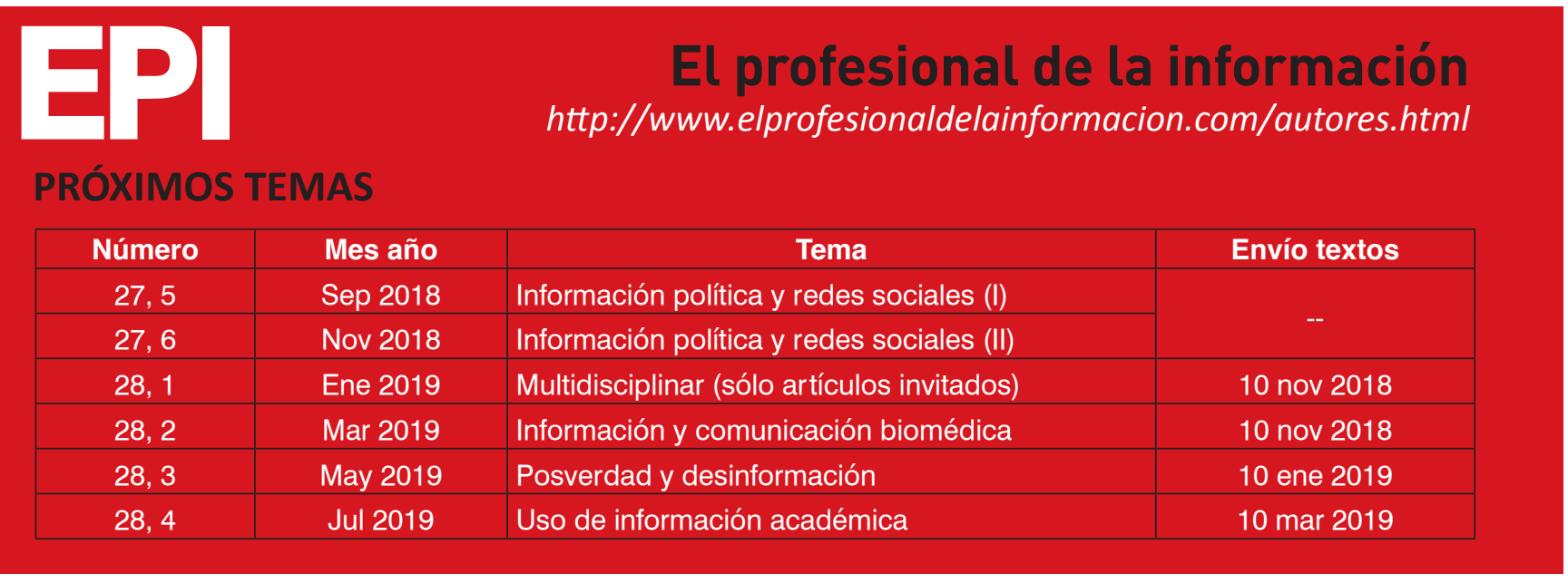

